\title{
THE INVERSE LIMIT AND FIRST DERIVED FUNCTOR, A SHORT EXACT SEQUENCE
}

\author{
GERALD LIEBERMAN
}

ABSTRACT. This paper provides a new topological proof that a certain sequence involving the inverse limit and first derived functor is short exact.

1. Introduction. The purpose of this paper is to provide a direct topological proof that the following is a short exact sequence:

$$
0 \rightarrow \operatorname{proj} \lim ^{1}\left[S Y^{n}, X\right] \rightarrow[Y, X] \rightarrow \operatorname{proj} \lim \left[Y^{n}, X\right] \rightarrow 0 \text {. }
$$

For the purposes of this paper we will restrict ourselves to the category of pointed sets. The inverse system used above is that induced from the inclusion of the $n$-skeleton $Y^{n}$ into $Y^{n+1}$ and proj $\lim ^{1}$ denotes the first derived functor. This fact is known and it or similar statements appear in a number of places in the literature. For example, Gray [3] states the result without proof, while Milnor [4] proves similar statements for homology and cohomology, providing the idea for the proof presented here. One could also arrive at this result as a simple application of Theorem 5.3 of Eckmann and Hilton [2]. However, there does not appear to be a direct proof in the literature. All spaces will be connected and of the homotopy type of CW-complexes. All maps and homotopies will be basepoint preserving.

2. Preliminaries. The notion of a cokernel will be needed to define the first derived functor. Cokernels exist in any category that has zero maps. We work in the category of pointed sets, and cokernels exist in this category.

Definition 1. Let $\mathcal{C}$ be a category with a zero morphism and let $X$ and $Y$ be objects in $\mathcal{C}$. Let $f$ be a morphism from $X$ to $Y$. The cokernel of $f$ is an object $Z$ of $\mathcal{C}$ and a morphism $q$ from $Y$ to $Z$ such that $q \circ F$ is the zero morphism and $q$ and $Z$ are universal with respect to this property.

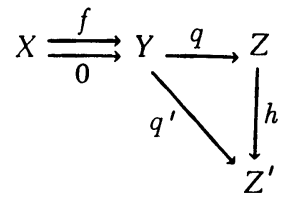

Received by the editors September 21, 1973.

AMS (MOS) subject classifications (1970). Primary 55E05; Secondary 55D 45.

Key words and phrases. Inverse limit, first derived functor, homotopy classes of maps, $H$-spaces. 
Let $f: X \rightarrow Y$ in the category of pointed sets. The cokernel of $f$ turns out to be $Y / f(X)$. That is, all points in $Y$ of the form $f(x)$ are identified with the basepoint.

Definition 2. Let $\left\{G_{n}, p_{n}\right\}$ be an inverse system of pointed sets. Define the inverse limit of $\left\{G_{n}, p_{n}\right\}$, denoted proj $\lim G_{n}$, as $\left\{\Pi_{n} g_{n} \in \Pi_{n} G_{n}: g_{n}=\right.$ $\left.p_{n+1}\left(g_{n+1}\right)\right\}$. If $\left\{G_{n}, p_{n}\right\}$ is an inverse system of groups, define $u: \Pi_{n} G_{n} \rightarrow$ $\Pi_{n} G_{n}$ by $u\left(\Pi_{n} g_{n}\right)=\Pi_{n}\left(g_{n} \cdot p_{n+1}\left(g_{n+1}\right)^{-1}\right)$. The first derived functor of the inverse limit of $\left\{G_{n}, p_{n}\right\}$, denoted proj $\lim ^{1} G_{n}$, is defined as the cokernel of $u$ in the category of pointed sets.

We remark here that proj $\lim ^{1}$ even changes its underlying set if one passes to the category of abelian groups.

For our proof, we follow Milnor [4] and define $L=\bigcup_{n \geq 1} Y^{n} \times[n-1, n] / \sim$, where $\sim$ identifies $(x, n-1) \in Y^{n} \times[n-1, n]$ and $(x, n-1) \in Y^{n-1} \times$ $[n-2, n-1]$ and $\sim$ also identifies $(*, i)$ and $(*, 0)$. The procedure is to construct a Mayer-Vietoris type argument using the excisive couple $\left(L_{1}, L_{2}\right)$ where $L_{1}=\bigcup_{n \text { odd }} Y^{n} \times[n-1, n] / \sim$ and $L_{2}=\bigcup_{n \text { even }} Y^{n} \times[n-1, n] / \sim$ are subspaces of $L$. Note that $h: L \rightarrow Y$ by $h(y, i)=y$ is a homotopy equivalence. Pictorially $L$ is:

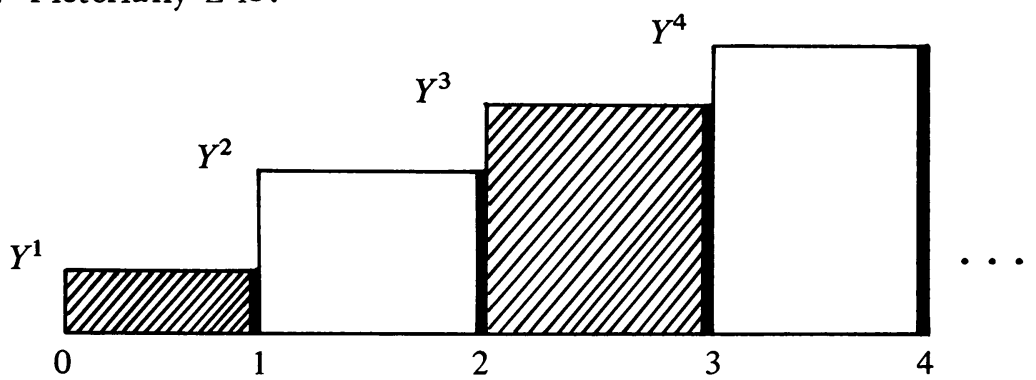

$L_{1}$ is the shaded portion, $L_{2}$ is the unshaded portion, and $L_{1} \cap L_{2}$ is the darkened portion. Since $L \simeq Y, L_{1} \simeq \bigvee_{n \text { odd }} Y^{n}, L_{2} \simeq \bigvee_{n \text { even }} Y^{n}$, and $L_{1} \cap L_{2} \simeq V_{n \geq 1} Y^{n}$, the following is obvious:

Proposition 3. (1) $\left[L_{1} \cap L_{2}, X\right] \approx \Pi_{n}\left[Y^{n}, X\right]$;

(2) $\left[L_{1}, X\right] \approx \Pi_{n \text { odd }}\left[Y^{n}, X\right]$;

(3) $\left[L_{2}, X\right] \approx \Pi_{n \text { even }}\left[Y^{n}, X\right]$;

(4) $[L, X] \approx[Y, X]$.

We need the dual version of Barratt and Whitehead's Lemma 7.4 [1] for a homotopy version of Mayer-Vietoris.

Lemma 4. Suppose that the following is a commutative diagram of groups and homomorphisms with exact rows: 


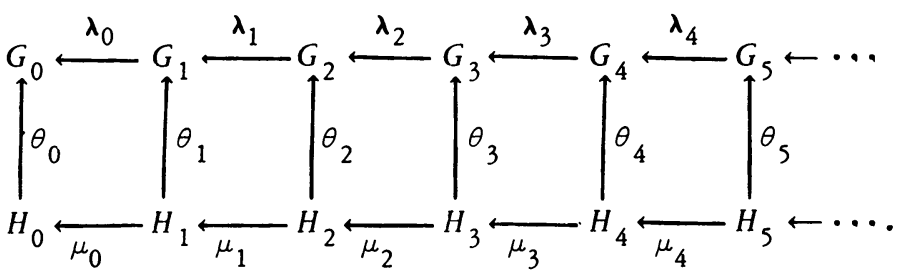

Suppose further that $\theta_{0}$ is monic and that $\theta_{3 k}$ is an isomorphism for all $k=1,2,3, \cdots$. Then,

$$
\begin{aligned}
& G_{1} \stackrel{u}{\longleftarrow} G_{2} \times H_{1} \stackrel{v}{\longleftarrow} H_{2} \stackrel{w}{\longleftarrow} G_{4} \leftarrow \cdots \leftarrow G_{i} \\
& \stackrel{u}{\longleftarrow} G_{i+1} \times H_{i} \stackrel{v}{\longleftarrow} H_{i+1} \stackrel{w}{\longleftarrow} G_{i+3} \leftarrow \cdots
\end{aligned}
$$

is exact where $i \equiv 1(\bmod 3), u=\lambda_{i} \cdot \theta_{i}^{-1}, v=\left(\theta_{i+1}, \mu_{i}\right)$, and $w=\mu_{i+1}$ 。 $\theta_{i+2}^{-1} \circ \lambda_{i+2}$. Moreover, if $G_{i}$ and $H_{i}$ are just pointed sets for $i=1,2$, and 3 (and need not exist for $i=0$ ), then the above sequence is still exact if terminated at $G_{2} \times H_{1} \stackrel{v}{\leftarrow} H_{2} \stackrel{w}{\leftarrow} \cdots$.

Proof. Since the diagram commutes and its rows are exact, image $v$ $\subset$ kernel $u$, image $w \subset$ kernel $v$, and image $u \subset$ kernel $w$. The reverse inclusions are all simple diagram chases. Note that if the groups fail to be abelian, $u$ need not be a homomorphism. Q.E.D.

Consider $L_{1} \cap L_{2} \hookrightarrow L_{1} \rightarrow L_{1} \cup c\left(L_{1} \cap L_{2}\right)$ and $L_{2} \hookrightarrow L=L_{1} \cup$ $L_{2} / \sim \rightarrow L \cup c\left(L_{2}\right)$. Then $L_{1} \cup c\left(L_{1} \cap L_{2}\right) \simeq L \cup c\left(L_{2}\right)$ since $\left(L_{1}, L_{2}\right)$ is an excisive couple. The Puppe sequences

$$
L_{1} \cap L_{2} \stackrel{i}{\longrightarrow} L_{1} \stackrel{j}{\longrightarrow} L_{1} \cup c\left(L_{1} \cap L_{2}\right) \stackrel{\delta}{\longrightarrow} S\left(L_{1} \cap L_{2}\right) \stackrel{S i}{\longrightarrow} S L_{1} \rightarrow \ldots
$$

and

$$
L_{2} \stackrel{i^{\prime}}{\longrightarrow} L \stackrel{j^{\prime}}{\longrightarrow} L \cup c\left(L_{2}\right) \stackrel{\delta^{\prime}}{\longrightarrow} S L_{2} \stackrel{S i^{\prime}}{\longrightarrow} S L \rightarrow \ldots
$$

yield the following commutative diagram with exact rows:

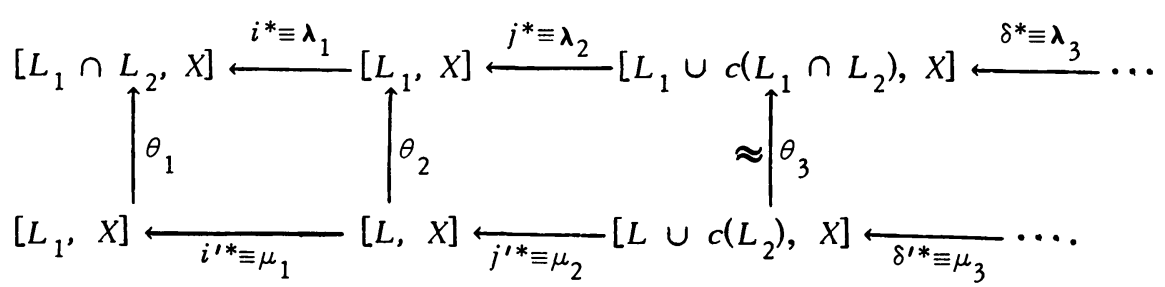

The $\theta_{i}$ are induced by the appropriate inclusion maps and $\theta_{3 k}$ is an isomorphism for $k=1,2, \cdots$. This is the situation required for Lemma 4 . 


\section{The exact sequence.}

\section{Theorem 5 .}

$$
0 \rightarrow \operatorname{proj} \lim ^{1}\left[S Y^{n}, X\right] \stackrel{w}{\longrightarrow}[Y, X] \stackrel{v}{\longrightarrow} \operatorname{proj} \lim \left[Y^{n}, X\right] \rightarrow 0
$$

is exact as a sequence of pointed sets, and is natural in the second factor. Moreover, if either $Y$ is a co-H-group or $X$ is a homotopy associative $H$-space, then whenever $f, g: Y \rightarrow X$ are such that $v([f])=v([g]),[f] \circ[g]^{-1} \in$ image $w$.

(We should remark that the moreover part of the theorem merely needs a natural group structure on $[Y, X]$.)

Proof. The above permits the application of Lemma 4 to conclude that

$$
\begin{array}{r}
{\left[L_{1}, X\right] \times\left[L_{2}, X\right] \stackrel{v}{\stackrel{u}{\longleftarrow}}[L, X] \stackrel{w}{\stackrel{u}{\longleftarrow}}\left[S L_{1}, X\right] \times\left[S L_{2}, X\right] \leftarrow \cdots}
\end{array}
$$

is exact. Remembering what $L_{1}, L_{2}, L$, and $L_{1} \cap L_{2}$ are yields via Proposition 3 the exact sequence

$$
\prod_{n}\left[Y^{n}, X\right] \stackrel{v}{\longleftarrow}[Y, X] \stackrel{w}{\longleftarrow} \prod_{n}\left[S Y^{n}, X\right] \stackrel{u}{\longleftarrow} \prod_{n}\left[S Y^{n}, X\right] \leftarrow \cdots
$$

Recall that $u: \Pi_{n}\left[S Y^{n}, X\right] \rightarrow \Pi_{n}\left[S Y^{n}, X\right]$ comes from

$$
u:\left[S L_{1}, X\right] \times\left[S L_{2}, X\right] \rightarrow\left[S\left(L_{1} \cap L_{2}\right), X\right]
$$

where $u([g],[h])=\lambda_{4}([g]) \cdot \theta_{4}\left([h]^{-1}\right)$. Hence, if $\Pi_{n}\left[g_{n}\right] \in \Pi_{n}\left[S Y^{n}, X\right]$, then $u\left(\Pi_{n}\left[g_{n}\right]\right)=\Pi_{n}\left(\left[g_{n}\right] \cdot\left[g_{n+1} \circ l\right]^{-1}\right)$ where $l$ is simply the inclusion of the skeletons, e.g., $S Y^{n} \rightarrow S Y^{n+1}$. Thus, ker $u=\operatorname{proj} \lim \left[S Y^{n}, X\right]$ and coker $u=$ proj $\lim ^{1}\left[S Y^{n}, X\right]$. Hence, $0 \rightarrow$ proj $\lim ^{1}\left[S Y^{n}, X\right] \stackrel{w}{\rightarrow}[Y, X] \stackrel{v}{\rightarrow}$ image $v \rightarrow$ 0 is exact as pointed sets. The basepoint of $[Y, X]$ is the class of the constant map to the basepoint of $X$, the basepoint of image $v$ is the product of the classes of the appropriate constant maps, and the basepoint of proj $\lim ^{1}\left[S Y^{n}, X\right]$ is the class of the product of the classes of the appropriate constant maps.

To complete the first half of the proof we merely have to identify image $v$ with proj $\lim \left[Y^{n}, X\right]$. Recall that image $v \subset\left[L_{1}, X\right] \times\left[L_{2}, X\right]$ consists of all ([g], $[h])$ where $g: L_{1} \rightarrow X, h: L_{2} \rightarrow X$ and $([g],[b])=v([d])$ for some $d: Y \rightarrow X$. Now, $v([d])=\left(\mu_{1}([d]), \theta_{2}([d])\right)=\left(\left[d \circ i^{\prime}\right],[d \circ k]\right)$, where $i^{\prime}: L_{2} \rightarrow$ $L$ and $k: L_{1} \rightarrow L$ are the inclusions. Thus, using the identifications of Proposition 3, $v([d])=([g],[h]) \in\left[L_{1}, X\right] \times\left[L_{2}, X\right]$ gets identified to $\Pi_{n}\left[d \mid Y^{n}\right] \in \Pi_{n}\left[Y^{n}, X\right]$. This is certainly in proj $\lim \left[Y^{n}, X\right]$ since $\left(d \mid Y^{n}\right) \mid Y^{n-1} \simeq$ $d \mid Y^{n-1}$. Hence, image $v \subset \operatorname{proj} \lim \left[Y^{n}, X\right]$. For the other direction, suppose 
that $\Pi_{n}\left[f_{n}\right] \in$ proj $\lim \left[Y^{n}, X\right] \subset \Pi_{n}\left[Y^{n}, X\right]$. Then $f_{n} \mid Y^{n-1} \simeq f_{n-1}$ by some homotopy $H_{n-1}^{\prime}: Y^{n-1} \times I \rightarrow X$. This induces. $H_{n-1}: Y^{n-1} \times I /\{(*, t)\} \rightarrow$ $X$. Define $f: L \rightarrow X$ by $f(y, t)=H_{n}(y, t)$ if $n-1 \leq t \leq n$. Pictorially:

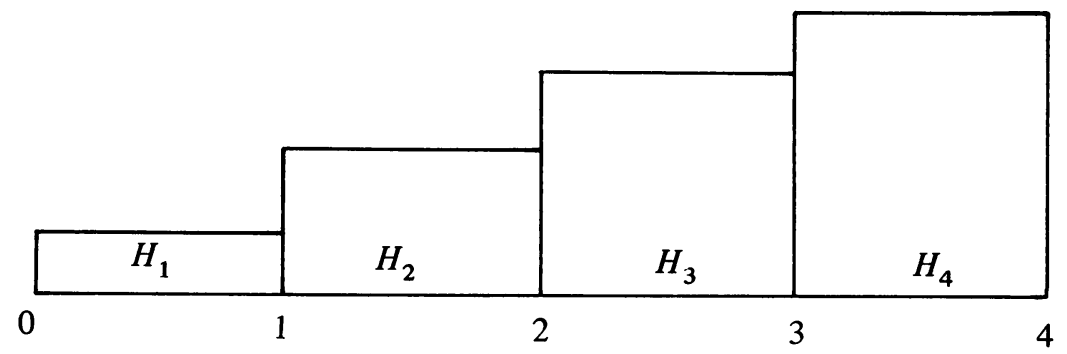

Now, $f\left|Y^{i} \times\{i\}=f_{i+1}\right| Y^{i} \simeq f_{i}$. Hence, $v([f])=\Pi_{n}\left[f_{n}\right]$, showing that image $v$ $=$ proj $\lim _{\iota}\left[Y^{n}, X\right]$. Since naturality is clear, the first half of the proof is now complete.

For the second statement, assume that either $Y$ is a co-H-group or that $X$ is a homotopy associative $H$-space. Then $[Y, X]$ and each $\left[Y^{n}, X\right]$ are groups $[5, \mathrm{p} .10]$, while each $\left[S Y^{n}, X\right]$ is an abelian group (if $Y$ is a co- $H^{\circ}$ group, assume that the comultiplication is cellular so that $Y^{n}$ is a co॰ ${ }^{\circ}$ group). Let $f, g: Y \rightarrow X$. In the sequence (*), all sets are now groups and all maps are homomorphisms. Suppose that $v([f])=v([g])$. Then

$$
v\left([f] \cdot[g]^{-1}\right)=v([f]) \cdot v([g])^{-1}=1 .
$$

Hence set exactness implies that there is an $a \in \operatorname{proj} \lim ^{1}\left[S Y^{n}, X\right]$ such that $w(a)=[f] \cdot[g]^{-1}$. Q.E.D.

\section{REFERENCES}

1. M. G. Barratt and J. H. C. Whitehead, The first nonvanishing group of an $(n+1)-a d$, Proc. London Math. Soc. (3) 6 (1956), 417-439. MR 19, 52.

2. B. Eckmann and P. J. Hilton, Unions and intersections in homotopy theory, Comment. Math. Helv. 38 (1964), 293-307. MR 29 \#5247.

3. B. I. Gray, Spaces of the same n-type, for all $n$, Topology 5 (1966), 241243. MR 33 \#4929.

4. J. Milnor, On axiomatic homology theory, Pacific J. Math. 12 (1962), 337341. MR 28 \#2544.

5. J. Stasheff, H-spaces from a homotopy point of view, Lecture Notes in Math., vol. 161, Springer-Verlag, Berlin and New York, 1970. MR 42 \#5261.

DEPARTMENT OF MATHEMATICS, UNIVERSITY OF ROCHESTER, ROCHESTER, NEW YORK 14627

DEPARTMENT OF MATHEMATICS, COLBY COLLEGE, WATERVILLE, MAINE 04901 (Current address) 